\title{
Congestion Control in Wireless Sensor Network based on Predicted Sensor Position on Movement for Body Area Network Applications
}

\author{
N. Thrimoorthy \\ M.Tech \\ Assistant Professor \\ New Horizon College of Engineering, \\ Bangalore, India.
}

\author{
T. Anuradha, $\mathrm{PhD}$ \\ Associate Professor, \\ Dept. of Computer Science, \\ Dravidian University, Kuppam,A.P.,India.
}

\begin{abstract}
WSN is a self organized network consisting of nodes. These nodes can have a small degree of movement due to medium on which they are deployed, like the sensor deployed on ocean water to detect conditions like tsunami. Since the nodes are moving, it is very difficult to maintain a reliable connection and when congestion happens in this network. Many congestion control protocols view only the current conditions and position of nodes while making the congestion control decisions and neglect the moving pattern of nodes. In this work we propose a congestion control protocol based on the movement pattern of nodes.
\end{abstract}

\section{Keywords}

WSN, WBAN, PCCP, ICD

\section{INTRODUCTION}

WSN are used in many applications like tsunami detection, ocean boarding alarming for fisherman. In this kind of applications due to nature of medium the sensors exhibit a small degree of movement. Wireless Body area networks (WBAN) are a typical case of this scenario. It is wireless network of wearable computing devices. WBAN devices may be embedded inside the body, implants, may be surfacemounted on the body in a fixed position Wearable technology or may be accompanied devices which humans can carry in different positions, in clothes pockets, by hand or in various bags. The sensors collects various parameters like glucose level, toxin levels and transfer via multi hop routing to the server systems where applications are available to act on sensor data.

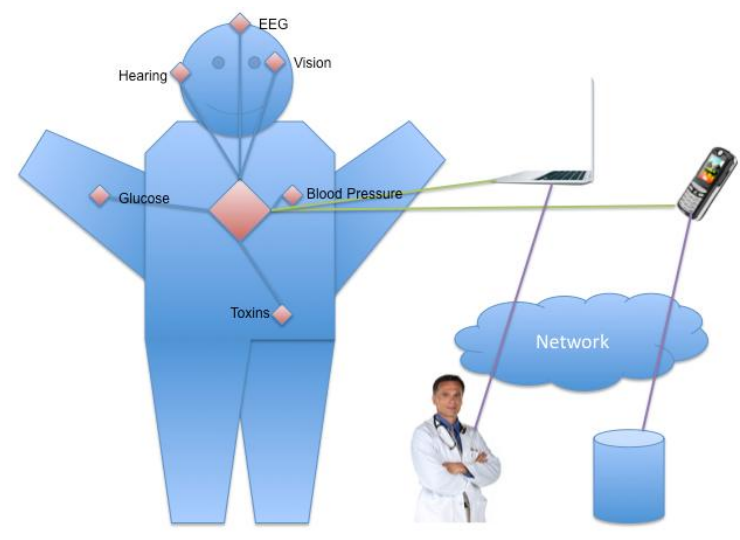

Fig.1: Body Area Networks

The popular IEEE 802.11 "WI-FI" protocol is capable of providing ad-hoc network facilities at low level, when no access point is available. In this network nodes establish connection with other nodes though multi hop and due to movement of nodes, the routing path is not always same. Since the routing path is not fixed, congestion control is also challenging in this network. The data is mostly communicated using store and forward because of intermittent connection failures. Reservation cannot be done, since path is not fixed for session duration. TCP based end to end congestion control is not so effective due to node movement.

Previously many studies have been made on congestion control in wireless sensor networks. Studies proposed solutions in different layers like physical, network and transport layers and also cross layers to detect and reduce congestion. Previous works can be classified into following types
1. Rate Based
2. Buffer Based
3. Priority Based
4. Cluster Based
5. Multipath routing based

Rate based algorithms estimates the number of flows from upstream nodes and modulates the rate of packet flow.

Buffer based algorithms tune their transmission rate and time based on the buffer occupancy of nodes in the routing flow.

Priority based algorithms assigns different priorities to flow and make their forwarding decisions based on the priority.

Cluster based algorithms decentralize the congestion control to are of scope by clustering the network and manage congestion.

Multi path routing algorithms divides and forwards packets across multiple path to reduce the congestion.

Even though nodes are having small degree of movement, the mobility is not completely random. In most deployments of this kind of sensor network, there is a pattern in the movement of nodes. They visit some places very often than other places and they meet some nodes more often than others. No previous congestion control mechanisms used this behavior and in this work we explore this behavior for congestion control. 


\section{RELATED WORK}

In this section we survey the current congestion detection and control protocols.

CODA [1] is an energy conserving and efficient control technique that is designed to solve congestion in the upstream direction i.e., the sensor to sink direction. It involves of two main schemes: 1) open loop hop by hop backpressure mechanism. 2) closed loop multisource regulation. The detection method in CODA is the receiver based congestion detection. It considers a combination of both present \& past loading conditions of the current buffer occupancy in the receiver. If the occupancy exceeds the threshold value, then congestion is inferred. The node detecting the congestion will notify its upstream neighbors to reduce the flow by backpressure mechanism. CODA detects congestion based on queue length and wireless channel loading. It uses AIMD rate adjustment technique and jointly used end-to-end and hop-byhop controls for regulation.

Event to Sink Reliable transport is a unique transport solution that is designed to achieve reliable event detection with minimum energy expenditure and congestion resolution [2]. This technique overcomes on of the disadvantages of CODA ESRT works based on two parameters: Event reliability and reporting frequency. Event reliability is defined as the number of data packets received at the decision interval at the sink. The end-to-end data delivery services are regulated by adjusting the sensor report frequency. If the reporting frequency is too low, the sink will not be able to collect enough information to detect the events. But on the other hand, if the reporting frequency is too high, it endangers the event transport reliability. ESRT adjusts the reporting frequency such that the observed event reliability is higher than the desired value to avoid congestion. The congestion detection in ESRT is by local buffer level of the sensors nodes. The sensor node adds a congestion notification bit on the packet's header when congestion occurs. When the sink receives this $\mathrm{CN}$ bit, it knows that congestion has happened in WSN.

Congestion control for Multiclass Traffic (COMUT) is a framework that consists of a distributed and scalable congestion control mechanism. It is based on selforganisation of networks into clusters. Each cluster is equipped with a sensor that is autonomously monitors congestion within its scope [3]. These networks are designed to support multiclass of traffic in WSN's. Each cluster is governed by a sentinel. These sentinel roles are assigned to sensors to proactively monitor the system and collect the event rates that is used to infer the combined level of congestion. The local traffic is reported by the sensors to the sentinel en-route a local broadcast system. The sensor rates per cluster are regulated by exchanging only small amounts of control information via regulator packets between the sentinel sensors alongside the flow path.

Congestion control for Sink to Sensors (CONSISE) [4] is a technique that works downstream i.e., from the sink to sensor direction. Conventionally, congestion happens in the sensorto-sink direction but, the reverse is also possible. The reasons are broadcast storm problem that refers to higher levels of collision that occurs on a series of local broadcast and reverse path traffic from sensors to sink. Congestion in the sensor-tosink direction will not be rare if WSN is built over CSMA/CA type of MAC and flooding based routing protocol.
Priority based congestion Control protocol (PCCP)[5] is an upstream congestion control protocol in WSN which measures congestion degree as the ratio of packet inter arrival time to the packet service time. It is designed in a way that the data packets have a guaranteed weighted fairness so that sink can get different throughput from the sensor nodes but in a weighted way. PCCP is intended to improve energy-efficient and support traditional QoS in terms of latency, throughput and packet loss ratio. PCCP can be of three components: 1) Intelligent Congestion Detection (ICD). 2) Implicit Congestion Notification (ICN). 3) Priority-based rate adjustment.

In $\mathrm{CCF}[6]$ algorithm each node measures the average rate $r$ at which packets can be sent from the node, divide the rate among the children nodes, adjust the rate if the queue is overloaded and propagate the rate downstream. It is designed to work with any MAC protocol in the data link layer and it exists in the transport layer. CCF uses packet service to deduce the availability of the service rate. It controls congestion in a hop-by-hop manner and each node uses exact rate adjustment based on its available service rate and child node number. It has two major disadvantages: The rate adjustment is based on packet service time which leads to low utilization as it has significant packet error rate. It cannot allocate the remaining effective capacity as it uses work conservation scheduling algorithm.

EB works in similar fashion to CODA. It uses congestion control in tree routing structure to all data sources to a sink. It uses the hop-by-hop backpressure mechanism. EB works in three steps: 1) each node calculates the average rate at which the data packets can be sent. 2) The node then divides the average data rate in to the number of children nodes to give the per-node data packet generation rate and adjusts the rate if the buffer is overflowing. 3) The node then compares the data rate of two children nodes with the parent nodes. The smaller rate among the two values is propagated such that data sources do not send packets beyond the minimum rate supported by the nodes along the path to the sink.

SenTCP[7] is a transport protocol that uses open loop hop-byhop Congestion Control. It has two distinct features that it adopts while detection. It detects congestion using local Congestion degree and uses hopby-hop for control[4]. The features include: 1) SenTCP conjointly uses average local packet service and average local packet inter-arrival time. These features determine the current local congestion degree in each intermediate sensor nodes. They effectively help to differentiate the reasons for packet loss and delay in wireless communication. 2) Each intermediate node issues a feedback signal backward and hop-by-hop control. This signal carries buffer occupancy ratio and local congestion degree. These parameters are used to adjust the sending rate of the neighbouring nodes in the transport layer[1],[10]. SenTCP realizes higher throughput and good energy efficiency since it reduces packet dropping by hop-by-hop. The major disadvantage of SenTCP is that it guarantees no reliability.

Pump Slowly and Fetch Quickly (PSFQ) [8] control protocol aims at distributing data from sink-to-sensors i.e., it belongs to the downstream reliability guarantee. PSFQ is a mechanism that is proposed for reprogramming a group of sensors. PSFQ is based on slowly injecting packets into the network "pump operation" and performing aggressive hop-by-hop recovery in case of packet loss "fetch operation" and selective status reporting "reporting operation". The disadvantages of PSFQ 
include: 1) Since it uses hop-by-hop recovery, it requires more buffer space. 2) The transmission of data packets are relatively slow in operation and hence there is large delay in the system. 3) PSFQ cannot detect a loss of single packets individually as it uses NACK signals for indication and the entire block is re transmitted upon request. 4) It cannot be used in the forward direction and does not address packet loss due to congestion.

\section{PROBLEM DEFINITION}

Given a sensor network, whose nodes moves frequently to any position in the network and movement is governed by a under laying pattern and any node wants to communicate with any other node in the network, the objective of work is reduce the congestion in the network. The congestion is measured in terms of failed packet transmission and interference ratio.

\section{A MOVEMENT PATTERN BASED CONGESTION CONTROL}

In mobile sensor network, every node moves but remains connected in network. A node is set to meet another node if both nodes are in communication. Every node meets another node with a contact frequency. Contact frequency is measured in terms of number of times the nodes meets another node over a period of time. The contact frequency is the reflection of how often the nodes meet. Higher value of contact frequency means nodes meet more often.

In this network, node can communicate with another node in a store and forward manner. Each intermediate node between source and target node acts a router relaying the packet. The storage available for packet buffering is limited and when more packets arrive than buffer space, the packets are dropped. Also if two or more nodes try to communicate in the same communication range again interference occurs. Due to the interference node reattempts and the intended communication nodes may be moved far way. This too results in packet delivery failure. In this network, as the packet life time in the buffer increases and if the interference is higher, there is more congestion in the network.

Congestion management is done using the contact frequency in the proposed solution. Before congestion management, the routing protocol over which the proposed congestion control operates is explained below.

Each node meets another node with a contact frequency. If the contact frequency is above certain threshold, then the nodes are in one Group. Among the nodes in the group, node which meets other nodes in the group more often is chosen as Group head, the node which meets other group nodes more often is chosen as Group ambassador.

The routing is split to two categories inter group and intra group.

When a node wants to communicate with other node in same group, it sends the data to the group head when it meets it and the group head sends the data to other node when it meets it. Since group head has good contact frequency with all nodes in the group, this communication is possible.

When a node wants to communicate with other node in other group, the node sends the data to group head, group head sends data to the corresponding group ambassador and the group ambassador deliver directly to node when it meets or to that group head which deliver data when it meet the node.
Above routing protocol works best when the storage space is able to meet the arrival rate without drops. When the arrival rate exceeds the storage space, congestion happens. Our method for reducing congestion based on contact frequency using following heuristics

1. Try delivery of data to low contact frequency target nodes ahead of high contact frequency target nodes. By this mechanism the packet queuing in buffer space is reduced and thereby congestion will be reduced. Packets stored in buffer space for high frequency targets can be scheduled at time since the chance of meeting is high, there packet buffer space can be reduced at will.

2. The data transfer rate is split across interfering nodes. By this way the completion for transfer is slotted fairly thereby reducing congestion due to interference.

The packets arrived at group head or at group ambassador is queued in buffer space. When a group node meets other nodes, it orders the meet nodes with increasing order of contact frequency and checks in the ordered meet nodes whether any packet is available and picks the packet and places in that order for delivery.

Whenever node moves to a new position or every time once it advertises a HELLO packet. In the Hello Packet, information about queue size is filled.

The total size of queue, the number of low contact frequency packets is present in the Hello Packet. Each node is thereby aware of its neighboring node data transfer requirement. The data transfer slot is split 3 parts. 1 part is reserved for all nodes with data to send and 2 parts is split proportionately to the low contact frequency packet count. By this way, low contact frequency packet is given more chance to be expelled from packet queues. If a node has received slot for low contact frequency, but that node is not in neighborhood, then the node can use the slots for high contact frequency packets.

\section{ANALYSIS}

We study the packet loss and packet delay in the proposed approach in this section. Let there be $\mathrm{N}$ nodes in the network. Each node $i$ has contact frequency with node $\mathrm{j}$ as $\mathrm{Cij}$. The threshold for making a group is $\alpha$ and two nodes are part of group when $\mathrm{Cij}>\alpha$. The frequency of meet up of group nodes be bound by maximum value say $\beta$ and the queue size is each node is say $¥$.

Let there be $\mathrm{M}$ groups in between a source node to sink, the maximum delay (D) for the packet to travel from source node to sink will be

$\mathrm{D}=\beta^{*} \mathrm{M}$

The delay is bound by the frequency of meet up of groups in between source and sink.

The proposed solution use opportunistic forwarding, so if there are $\mathrm{M}$ groups in between source and sink, the packet may loss if any of $\mathrm{M}$ group leader node $\mathrm{Q}$ is not empty when the hop group nodes meet up. The Q not empty is controlled by the frequency of meet up $\beta$, if the all messages in $Q$ are intended for different group hops and their frequency of meet up is delayed, there is more chance of the message being lost, if further messages is generated from source. 


\section{RESULTS}

To test the performance of proposed solution we implemented the solution on JProwler. The simulation was conducted with following parameters

\begin{tabular}{|l|l|}
\hline Parameters & Values \\
\hline Number of Nodes & 100 to 200 \\
\hline Communication range & $100 \mathrm{~m}$ \\
\hline Area of simulation & $1000 \mathrm{~m} * 1000 \mathrm{~m}$ \\
\hline Packet Rate & 10 to 40 packet per second \\
\hline Simulation time & 30 seconds \\
\hline Interface Queue Length & 50 \\
\hline MAC & 802.11 \\
\hline Movement Speed & $5 \mathrm{~m} / \mathrm{sec}$ \\
\hline
\end{tabular}

At each time interval of $5 \mathrm{sec}, 10$ nodes generated packet with rate of 10 to 40 as configured and maintained the traffic rate for $5 \mathrm{sec}$. The proposed solution is compared with priority based congestion control scheme mentioned in [5] and senTCP mentioned in [7]. We measured following parameters
1. Throughput
2. Packet Success ratio
3. Packet Delay
4. No of congestion points

Throughput is measured as the number of packets received at target nodes.

Packet success ratio is the ratio of number of packet received successfully at target nodes to the number of packets sent.

Packet delay is the end to end delay for packet traversal from source to target node.

A node is congestion point if its interface queue occupancy is more than $90 \%$.

Throughput is calculated by increasing the packet rate from 10 to 40 insteps of 5 and results are plotted below. From the results we see that proposed solution achieves better throughput than other solution.

\section{Throughput}

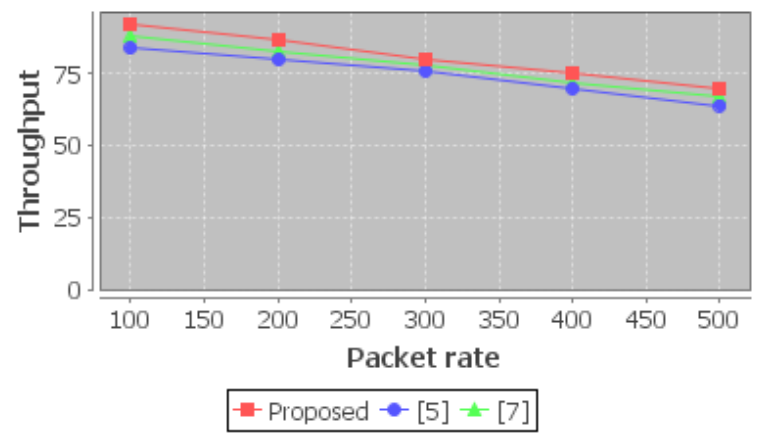

Packet success ratio is calculated by increasing the packet rate from 10 to 40 insteps of 5 and results are plotted below. From the results we see that proposed solution achieves better success ratio, the reason being the number of lost packets reduced due to better management of packet queues.

\section{Packet Success Ratio}

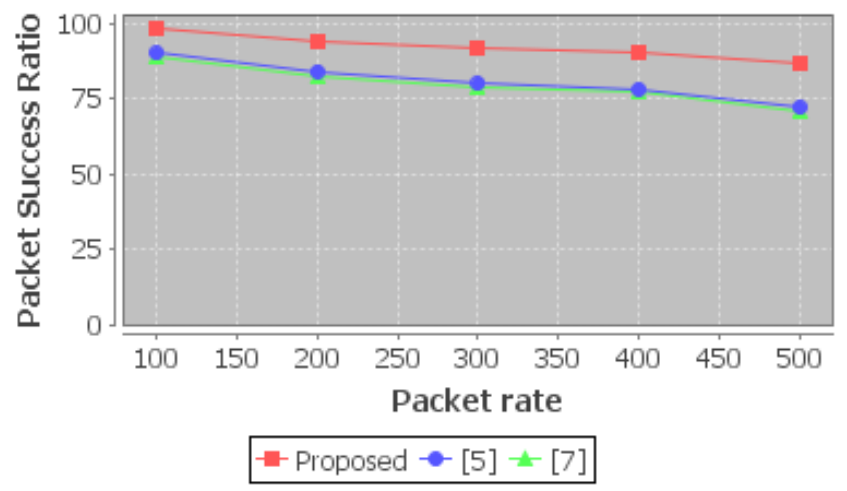

Packet delay is calculated by increasing the packet rate from 10 to 40 in steps of 5 and the results are plotted below. From the results we see that the proposed solution has comparatively higher delay the reason being queuing delay in nodes

\section{Delay}

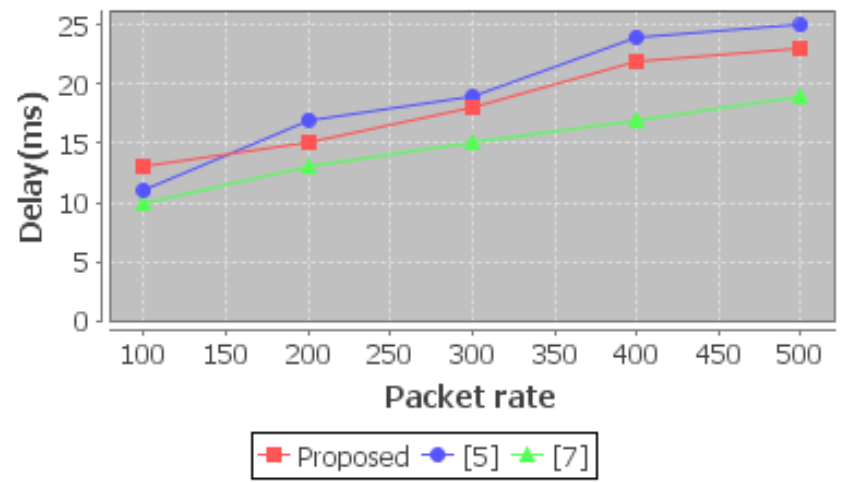

We measured the number of congestion points by varying the packet rate from 10 to 40 in steps of 5 and the results show that number of congestion points is very less in our proposed solution.

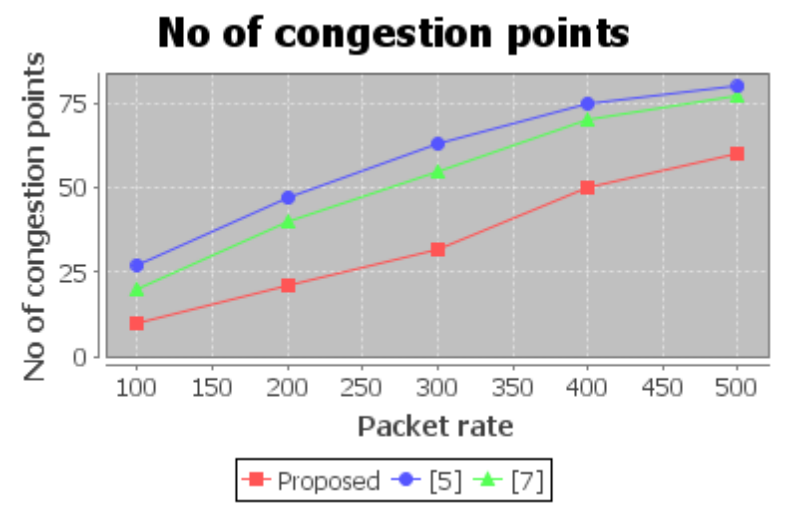

\section{CONCLUSION}

In this work, we have explained the movement based congestion control mechanism. Due to medium movement, the sensor position changes and by prediction of movement information and using it for opportunistic communication we have reduced the congestion in network. Through simulation 
we have proved that our proposed protocol has low congestion when compared to other congestion control algorithms for the case of body area networks. Due to congestion reduction packet loss is reduced and network throughput is increased. The accuracy of prediction can be still improved by applying other models like HMM, this can be explored as a part of future work.

\section{REFERENCES}

[1] Chien- Yin Wan, Shane B. Eiseaman, Andrew T. Champbell, " CODA: Congestion Detection and Avoidance".

[2] Ozgur B. Akan, Ian F. Akyildiz, "Event-to-Sink Reliable Transport in wireless Sensor Networks".IEEE/ACM transactions on Networking. Vol. 13, No.5, October 2005

[3] KyriakosKarenos, VanaKalogeraki, Srikanth V. Krishnamurthy. "Cluster-based Congestion control for sensor networks".

[4] RamujaVedantham, RaghupathySivakumar, Sueng Jong park, "Sink-to-Sensors Congestion Control Strategy".

[5] Mohammad HosseinYaghmaee, Donald Adjeroh. " A New priority Based Congestion Control Protocol for Wireless Multimedia Sensor Networks”. IEEE 2008.

[6] Swastil Brahma, MainakChatterjee, Kevin Kwiat," Congestion Control and Fairness in Wireless Sensor Networks"

[7] C. Wang, K.Shoraby, B.Li, "SenTCP: A hop-byhop Congestion Control protocol for Wireless Sensor Networks" in IEEE INFOCOM 2005, USA, March 2005.

[8] C. Y. Wan, A.T. Campbell, "PSFQ: A reliable transport protocol for Wireless Sensor Networks" in proceedings of ACM WSNA'02, September 28, 2002, USA.

[9] K.ChenandK.Nahrstedt. EXACT:AnExplicitRatebasedFlowControlFrameworkinMANET(extendedversio n). Technical Report UIUCDCS-R-2002-2286/UILU-
ENG-2002-1730, Department of Computer Science, University of Illinois at Urbana-Champaign, July 2002.

[10] K. Chen and K. Nahrstedt. Limitations of EquationBased Congestion Control in Mobile Ad Hoc Networks. In ICDCSW '04: Proceedings of the 24th International Conference on Distributed Computing Systems Workshops W7: EC, pages 756-761, 2004. DOI: 10.1109/ICDCSW.2004.1284118.

[11] K. Chen, K. Nahrstedt, and N. Vaidya. The Utility of Explicit Rate-based Flow Control in Mobile Ad Hoc Networks. In WCNC '04: Proceedings of the IEEE Wireless Communications and Networking Conference, volume 3, pages 1921-1926, Mar. 2004.

[12] K. Chen, Y. Xue, and K. Nahrstedt. On Setting TCP's Congestion Window Limit in Mobile Ad Hoc Networks. In ICC '03: Proceedings of the IEEE International Conference on Communications, Anchorage, Alaska, May 2003. DOI: 10.1109/ICC.2003.1204525.

[13] C. de M. Cordeiro, S. R. Das, and D. P. Agrawal. COPAS: Dynamic Contention-Balancing to Enhance the Performance of TCP over Multi-hop Wireless Networks. In Proceedings of the 10th International Conference on Computer Communication and Networks (IC3N), pages 382-387, Miami, FL, USA, Oct. 2002.

[14] R. de Oliveira and T. Braun. A Delay-based Approach Using Fuzzy Logic to Improve TCP Error Detection in Ad Hoc Networks. In WCNC '04: Proceedings of the IEEE Wireless Communications and Networking Conference, volume 3, pages 1666-1671, Mar. 2004.

[15] R. de Oliveira and T. Braun. A Dynamic Adaptive Acknowledgment Strategy for TCP over Multihop Wireless Networks. In INFOCOM '05: Proceedings of the 24th Annual Joint Conference of the IEEE Computer and Communications Societies, volume 3, pages 1863 1874, Mar. 2005.2 DOI: 10.1109/INFCOM.2005.1498465. 